Supporting Information for

\title{
MetDIA: Targeted Metabolite Extraction of Multiplexed MS/MS Spectra Generated by Data-Independent Acquisition
}

Hao Li, Yuping Cai, Yuan Guo, Fangfang Chen, and Zheng-Jiang Zhu*

Interdisciplinary Research Center on Biology and Chemistry, and Shanghai Institute of Organic Chemistry, Chinese Academy of Sciences, Shanghai, 200032 P.R. China

Corresponding Author

*E-mail: jiangzhu@sioc.ac.cn, phone: 86-21-68582296 


\section{File list in the zip file "Result output files" in the Supporting Information:}

csv file 01: MetDIA result for 30STD_mix 330ppb.csv

csv file 02: MetDIA result for 30STD_mix 33ppb.csv

csv file 03: MetDIA result for $13 \mathrm{C}$ fully labeled bacteria E.coli.csv

csv file 04: MetDIA result for human serum.csv

csv file 05: MetDIA result for bacteria E.coli.csv

csv file 06: MetDIA result for rat liver tissue

csv file 07: MetDIA result for swath Jurkat cell (FADD deficient vs wt)

csv file 08: DDA result for 30STD_mix 330ppb.csv

csv file 09: DDA result for 30STD_mix 33ppb.csv

csv file 10: DDA result for human serum.csv

csv file 11: DDA result for bacteria E.coli.csv

csv file 12: DDA result for rat liver tissue.csv

csv file 13: MS-DIAL result for 30STD_mix 330ppb.csv

csv file 14: MS-DIAL result for 30STD_mix 33ppb.csv

csv file 15: MS-DIAL result for human serum.csv

csv file 16: MS-DIAL result for bacteria E.coli.csv

csv file 17: MS-DIAL result for rat liver tissue.csv

csv file 18: Demo spectral database.csv

csv file 19: Detail information of 786 standards.csv

csv file 20: windows.csv

R file 1: Instruction for data processing.R

R file 2: Demo spectral database. $R$ 


\begin{tabular}{|c|c|c|c|c|c|}
\hline Name & LABID & EM & Formula & HMDB & KEGG \\
\hline Cytidine & L0013 & 243.0855 & $\mathrm{C} 9 \mathrm{H} 13 \mathrm{~N} 3 \mathrm{O} 5$ & HMDB00089 & $\mathrm{C} 00475$ \\
\hline D-Biotin & L0014 & 244.0882 & $\mathrm{C} 10 \mathrm{H} 16 \mathrm{~N} 2 \mathrm{O} 3 \mathrm{~S}$ & HMDB00030 & C00120 \\
\hline Adenosine & L0016 & 267.0968 & $\mathrm{C} 10 \mathrm{H} 13 \mathrm{~N} 5 \mathrm{O} 4$ & HMDB00050 & $\mathrm{C} 00212$ \\
\hline S-Adenosyl-L-homocysteine & L0040 & 384.1216 & $\mathrm{C} 14 \mathrm{H} 20 \mathrm{~N} 6 \mathrm{O} 5 \mathrm{~S}$ & HMDB00939 & $\mathrm{C} 00021$ \\
\hline Flavin adenine dinucleotide & L0043 & 785.1571 & $\mathrm{C} 27 \mathrm{H} 33 \mathrm{~N} 9015 \mathrm{P} 2$ & HMDB01248 & $\mathrm{C} 00016$ \\
\hline N-Acetylputrescine & L0083 & 130.1106 & $\mathrm{C} 6 \mathrm{H} 14 \mathrm{~N} 2 \mathrm{O}$ & HMDB02064 & $\mathrm{C} 02714$ \\
\hline Acetylcarnitine & L0133 & 203.1157 & $\mathrm{C} 9 \mathrm{H} 17 \mathrm{NO} 4$ & HMDB00201 & $\mathrm{C} 02571$ \\
\hline $\begin{array}{l}\text { 2'-Deoxyadenosine } \\
\text { 5'-monophosphate }\end{array}$ & L0140 & 331.0682 & $\mathrm{C} 10 \mathrm{H} 14 \mathrm{~N} 5 \mathrm{O} 6 \mathrm{P}$ & HMDB00905 & $\mathrm{C} 00360$ \\
\hline N2,N2-Dimethylguanosine & L0213 & 311.123 & C12H17N5O5 & HMDB04824 & N/A \\
\hline 3'-O-methylguanosine & L0235 & 297.1073 & C11H15N5O5 & HMDB06038 & N/A \\
\hline Guanidoacetic acid & S0005 & 117.0538 & $\mathrm{C} 3 \mathrm{H} 7 \mathrm{~N} 3 \mathrm{O} 2$ & HMDB00128 & $\mathrm{C} 00581$ \\
\hline Hypoxanthine & S0012 & 136.0385 & $\mathrm{C} 5 \mathrm{H} 4 \mathrm{~N} 4 \mathrm{O}$ & HMDB00157 & C00262 \\
\hline Inosine & S0032 & 268.0808 & $\mathrm{C} 10 \mathrm{H} 12 \mathrm{~N} 4 \mathrm{O} 5$ & HMDB00195 & C00294 \\
\hline 4-Aminobutyrate & S0039 & 103.0633 & C4H9NO2 & HMDB00112 & $\mathrm{C} 00334$ \\
\hline Creatinine & S0042 & 113.0589 & C4H7N3O & HMDB00562 & C00791 \\
\hline Creatine & S0047 & 131.0695 & $\mathrm{C} 4 \mathrm{H} 9 \mathrm{~N} 3 \mathrm{O} 2$ & HMDB00064 & $\mathrm{C} 00300$ \\
\hline Nicotinamide & S0048 & 122.048 & $\mathrm{C} 6 \mathrm{H} 6 \mathrm{~N} 2 \mathrm{O}$ & HMDB01406 & $\mathrm{C} 00153$ \\
\hline L-Pipecolic acid & S0050 & 129.079 & $\mathrm{C} 6 \mathrm{H} 11 \mathrm{NO} 2$ & HMDB00716 & $\mathrm{C} 00408$ \\
\hline L-Glutamine & S0055 & 146.0691 & $\mathrm{C} 5 \mathrm{H} 10 \mathrm{~N} 2 \mathrm{O} 3$ & HMDB00641 & C00064 \\
\hline L-Carnitine & S0060 & 161.1052 & $\mathrm{C} 7 \mathrm{H} 15 \mathrm{NO} 3$ & HMDB00062 & $\mathrm{C} 00318$ \\
\hline L-Citrulline & S0063 & 175.0957 & C6H13N3O3 & HMDB00904 & C00327 \\
\hline Guanosine & S0071 & 283.0917 & $\mathrm{C} 10 \mathrm{H} 13 \mathrm{~N} 5 \mathrm{O} 5$ & HMDB00133 & $\mathrm{C} 00387$ \\
\hline Riboflavin & S0072 & 376.1383 & $\mathrm{C} 17 \mathrm{H} 20 \mathrm{~N} 4 \mathrm{O} 6$ & HMDB00244 & $\mathrm{C} 00255$ \\
\hline Kynurenic acid & S0079 & 189.0426 & $\mathrm{C} 10 \mathrm{H} 7 \mathrm{NO} 3$ & HMDB00715 & C01717 \\
\hline Pyridoxine & S0080 & 169.0739 & $\mathrm{C} 8 \mathrm{H} 11 \mathrm{NO} 3$ & HMDB00239 & $\mathrm{C} 00314$ \\
\hline Glycerophosphocholine & S0106 & 257.1028 & $\mathrm{C} 8 \mathrm{H} 21 \mathrm{NO} 6 \mathrm{P}$ & HMDB00086 & $\mathrm{C} 00670$ \\
\hline Purine & S0108 & 120.0436 & $\mathrm{C} 5 \mathrm{H} 4 \mathrm{~N} 4$ & HMDB01366 & C15587 \\
\hline 2-Hydroxyadenine & S0183 & 151.0494 & C5H5N5O & HMDB00403 & $\mathrm{N} / \mathrm{A}$ \\
\hline DL-2-Aminooctanoic acid & S0189 & 159.1259 & $\mathrm{C} 8 \mathrm{H} 17 \mathrm{NO} 2$ & HMDB00991 & $\mathrm{N} / \mathrm{A}$ \\
\hline L-Histidinol & S0192 & 141.0902 & $\mathrm{C} 6 \mathrm{H} 11 \mathrm{~N} 3 \mathrm{O}$ & HMDB03431 & $\mathrm{C} 00860$ \\
\hline
\end{tabular}

Table S1. Detailed information of 30 metabolite standards in 30STD_mix sample. 


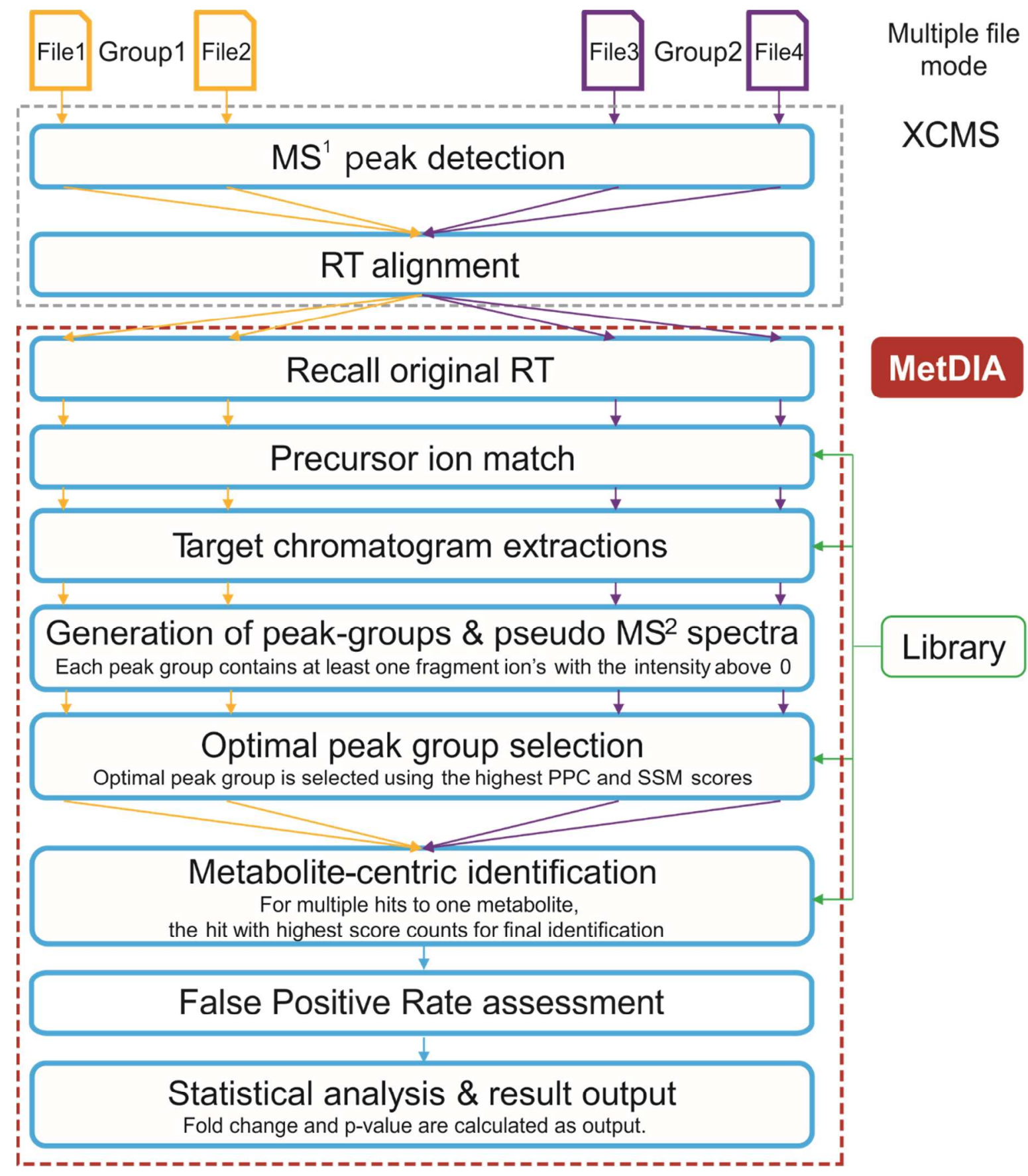

Scheme S1. The data processing of multiple files from different sample groups using MetDIA. 


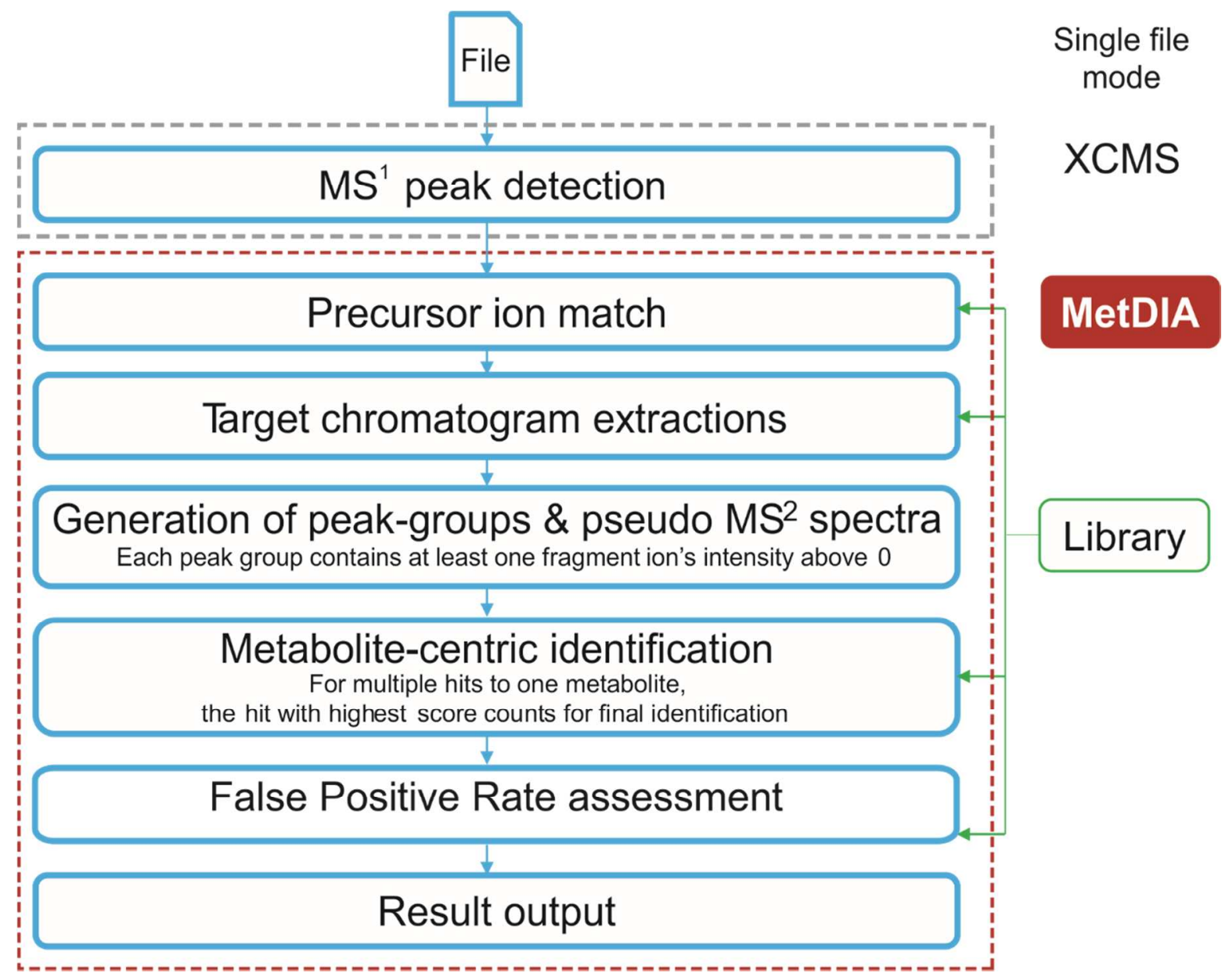

Scheme S2. The data processing of single file using MetDIA. 

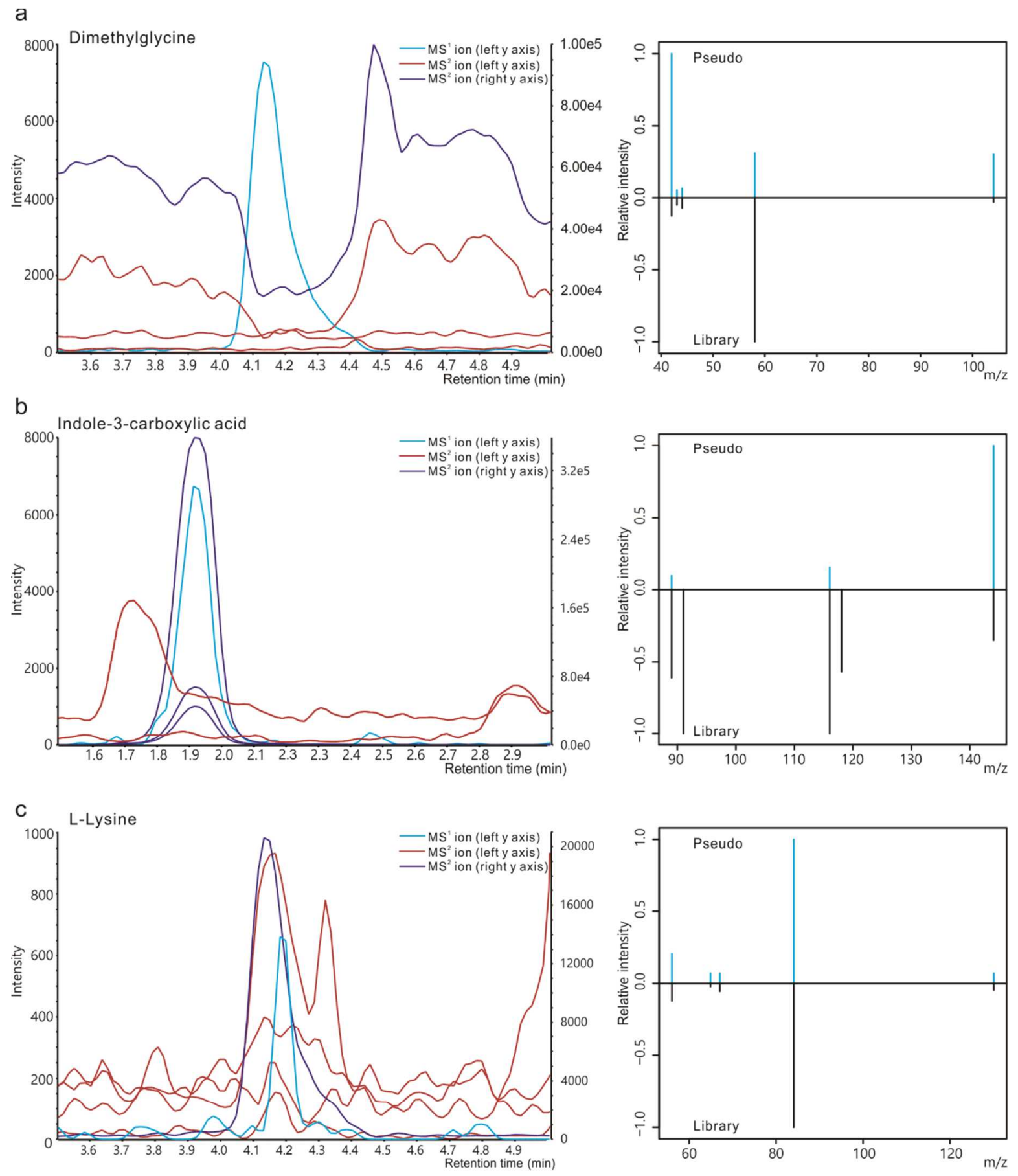

Figure S1. Examples of false positive identifications of metabolites in 30STD_mix sample: (a) true negative identification of dimethylglycine with low scores in both PPC (0.653) and SSM (0.407); (b) True negative identification of indole-3-carboxylic acid with high PPC score (0.985) but low SSM score (0.337); (c) true negative identification of L-lysine with low PPC score (0.7847) but high SSM score (0.995). 

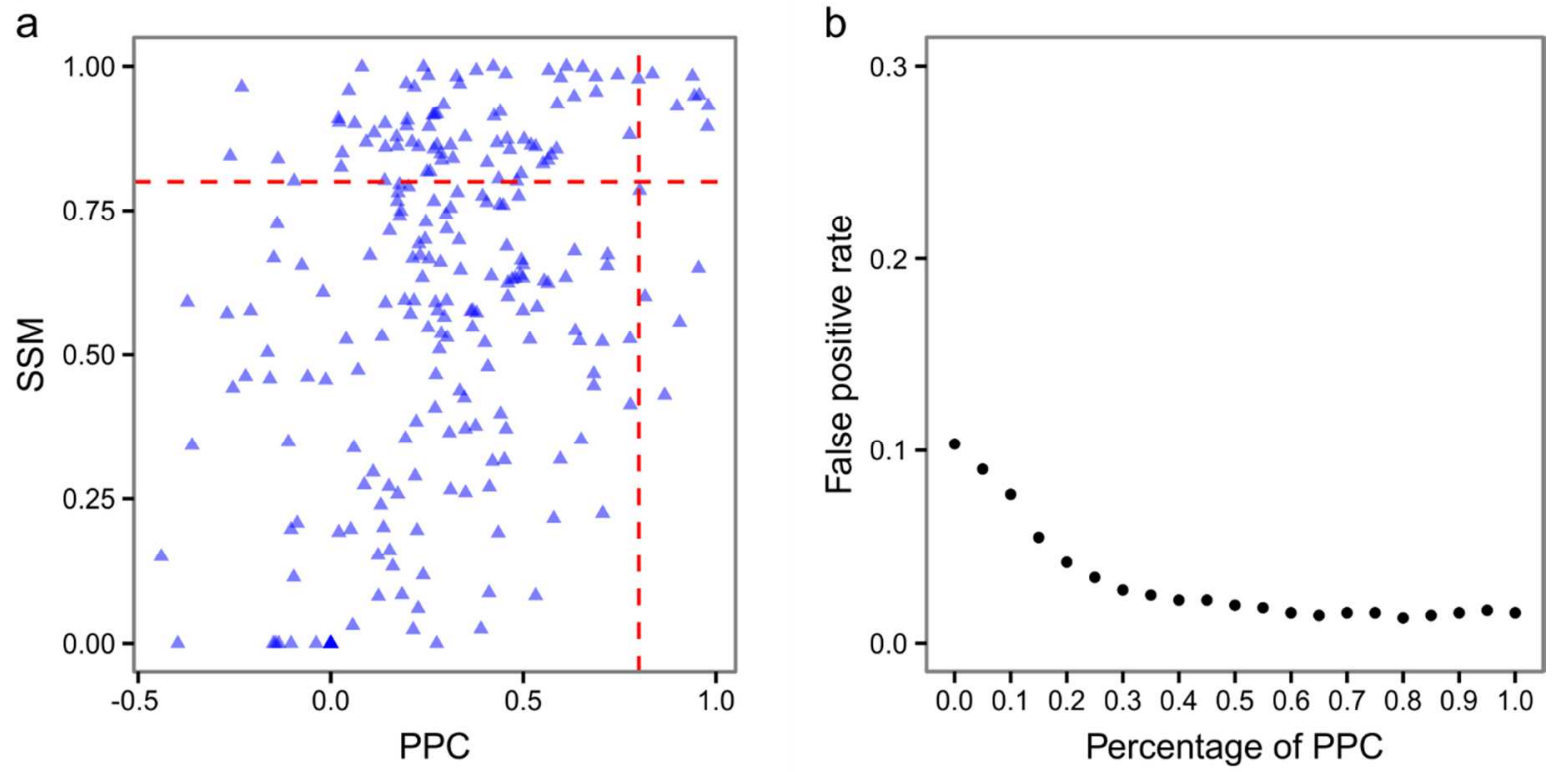

Figure S2. (a) The distributions of peak-peak correlation (PPC) scores and spectrum-spectrum match (SSM) scores for the metabolites extracted from ${ }^{13} \mathrm{C}$ fully labeled bacteria sample. (b) The false positive rate with different combination of PPC and SSM scores. 

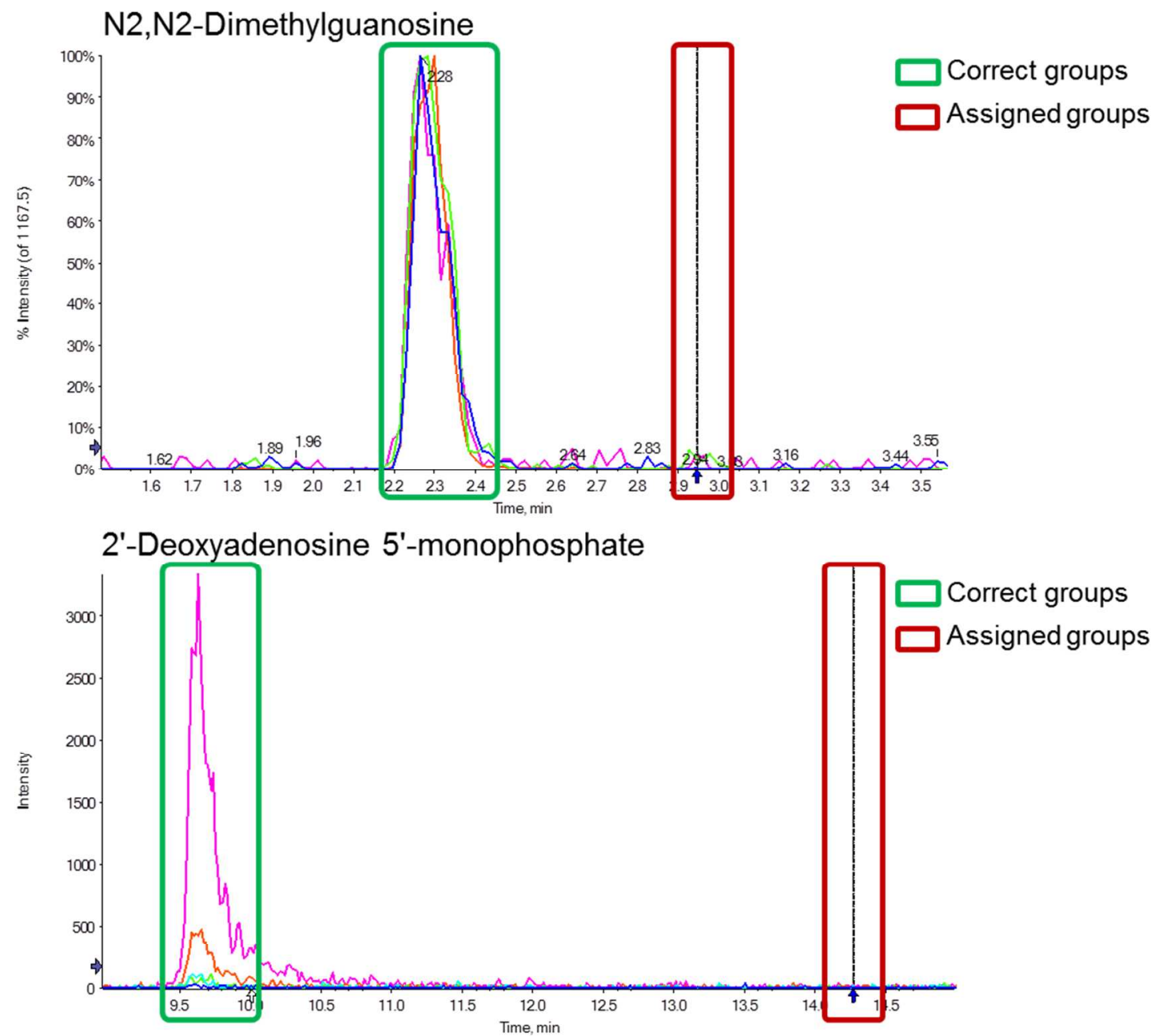

Figure S3. Two examples of mistakenly assigned peak groups extracted using AB Sciex SWATH ${ }^{\mathrm{TM}}$ (a software plugin in Peakview to process SWATH data). 

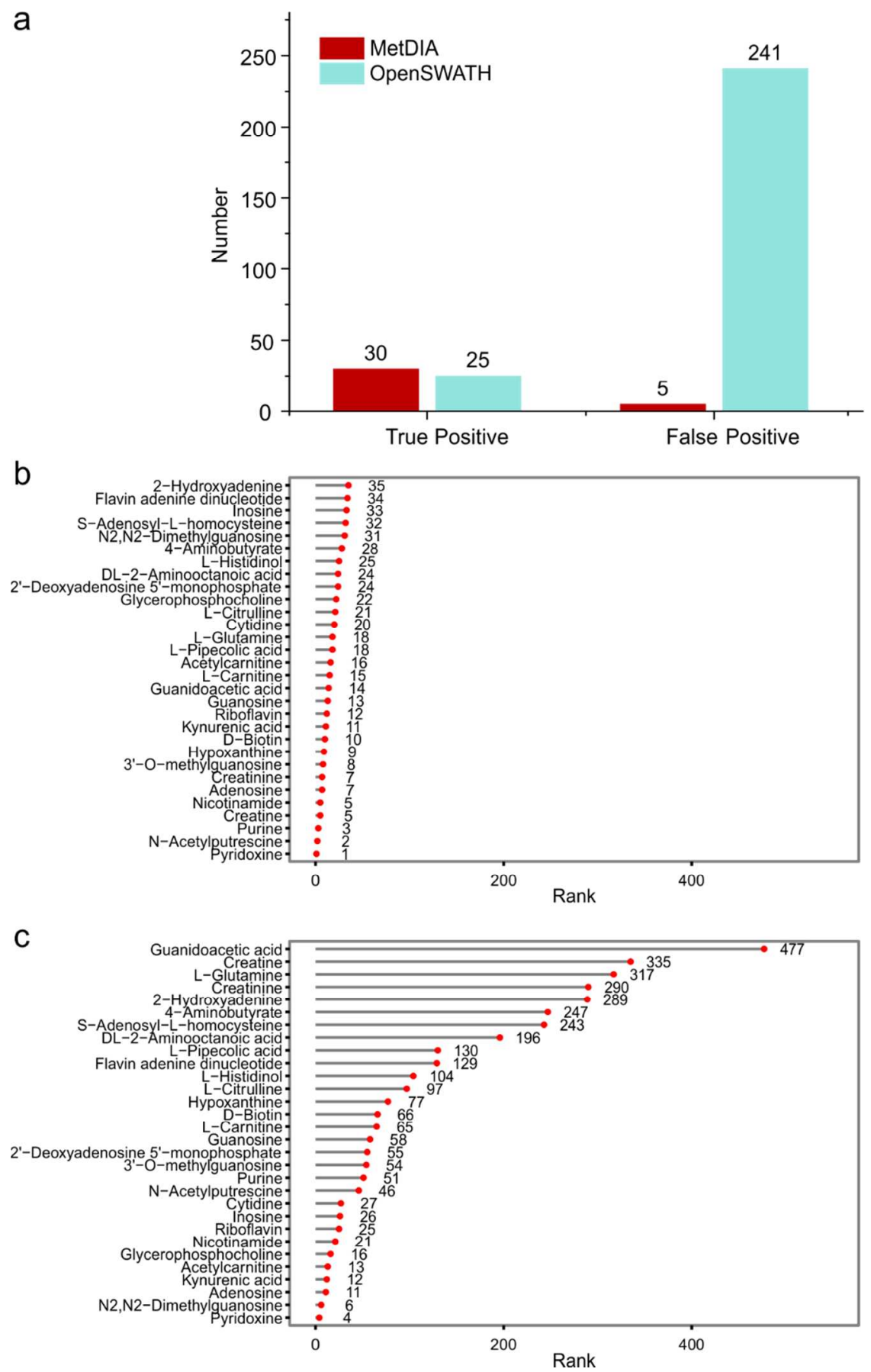

Figure S4. (a) Comparison of the true positive and false positive identifications in MetDIA and OpenSWATH with 30STD_mix samples. (b) The score ranking of 30 metabolites in MetDIA program. (c) The score ranking of 30 metabolites in OpenSWATH program. 


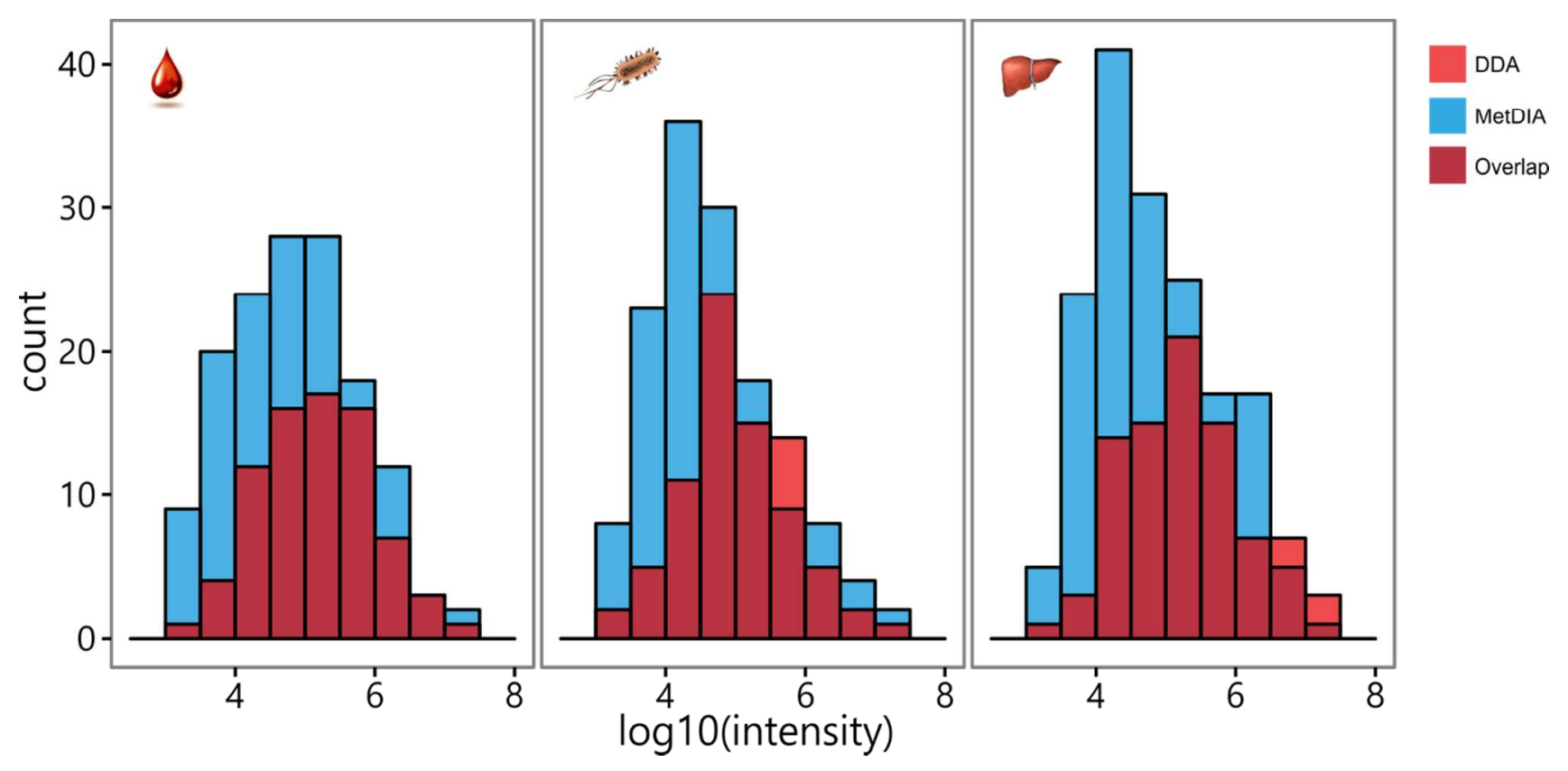

Figure S5. Comparison of intensity distribution of identified metabolites by MetDIA and DDA in biological samples (human serum, E.coli bacteria and rat liver tissue respectively). 


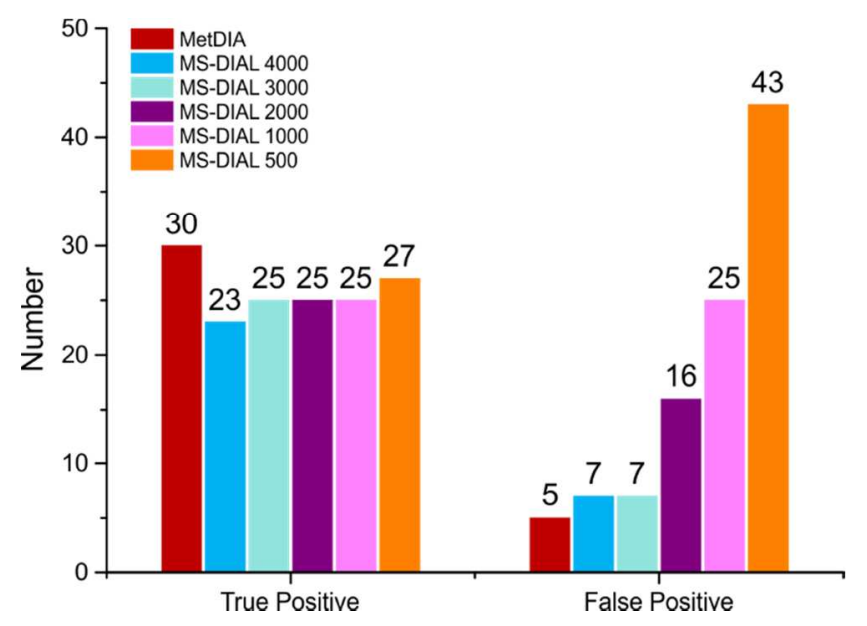

Figure S6. Optimization of "minimum peak height" (parameter of peak detection) in MS-DIAL, and comparison of identified and misidentified metabolites using MetDIA and MS-DIAL methods in 30STD_mix samples (3 replicates). Reducing "minimum peak height" provides more sensitive peak detection, but increases the false positive identifications. The parameter "minimum peak height" in MS-DIAL is set as 3000 for balanced the sensitivity and specificity. 


\section{Supplemental Experimental Section.}

Chemicals. Chemical standards were all purchased from Sigma Aldrich (St. Louis, MO, USA) and J\&K Scientific (Beijing, China). Ammonium acetate and ammonium hydroxide were purchased from Fisher Scientific (Morris Plains, NJ, USA). LC-MS grade water $\left(\mathrm{H}_{2} \mathrm{O}\right)$ and methanol $(\mathrm{MeOH})$ and acetonitrile (ACN) were purchased from Merck.

Metabolite extraction. The extraction of $E$. coli samples, human serum samples and Jurkat cell samples follows the protocol in our previous publication. ${ }^{1}$ Rat liver tissue $(\sim 10 \mathrm{mg})$ was first homogenized with ceramic beads and $200 \mu \mathrm{L}$ of $\mathrm{H}_{2} \mathrm{O}$ for three times using Precellys homogenizers, and each time homogenized for $20 \mathrm{~s}$ with $5 \mathrm{~s}$ intervals. After tissue homogenization, a volume of $800 \mu \mathrm{L}$ of $\mathrm{MeOH}: \mathrm{ACN} \quad(1: 1, \mathrm{v} / \mathrm{v})$ was added to samples as extraction solvent. The further metabolite extraction protocol was the same as the extraction of human serum samples. For metabolomic analyses of Jurkat cell samples, both groups (wide type and FADD deficient cells) have 18 biological replicates. For each replicate, $5 \times 10^{6}$ cells were harvested for the metabolite extraction.

LC-MS condition. Waters ACQUITY UPLC BEH Amide columns (particle size, $1.7 \mu \mathrm{m} ; 100 \mathrm{~mm}$ (length) $\times 2.1 \mathrm{~mm}$ (i.d.)) and Merck SeQuant ZIC-HILIC columns (particle size, $3.5 \mu \mathrm{m} ; 100 \mathrm{~mm}$ (length) $\times 2.1 \mathrm{~mm}$ (i.d.)) were used for the analyses. For ZIC-HILIC column, the mobile phases $A=10 \mathrm{mM}$ ammonium acetate in $95 \%$ water and $5 \%$ acetonitrile, and $B=10 \mathrm{mM}$ ammonium acetate in $5 \%$ water and $95 \%$ acetonitrile, were used for both in ESI positive and negative modes. And the linear gradient elutes from $99 \%$ B to $40 \%$ B (0-13 min), $40 \%$ B (13-15 min), $40 \%$ B to $99 \%$ B (15.0-15.1 min), then stays at $99 \% B$ for 4.9 minutes. For amide column, the mobile phases $A=25 \mathrm{mM}$ ammonium acetate and $25 \mathrm{mM}$ ammonium hydroxide in $100 \%$ water, and $\mathrm{B}=100 \%$ acetonitrile, were used for both in $\mathrm{ESI}$ positive and negative modes. And the linear gradient elutes from $85 \%$ B to $65 \%$ B (0.0-12.0 min), $65 \%$ $B$ to $40 \%$ B (12.0-12.1 min), $40 \%$ B (12.1-15.0 min), $40 \%$ B to $85 \%$ B (15.0-15.1 min), then stays at $85 \%$ $B$ for 4.9 minutes. The flow rate was $0.3 \mathrm{~mL} / \mathrm{min}$ and the sample injection volume was $2 \mu \mathrm{L}$. ESI source paratmeters on TripleTOF 5600 were set as followings: ion source gas 1 (GS1), 60 psi; ion source Gas 2 (GS2), $60 \mathrm{psi}$; curtain gas (CUR), $20 \mathrm{psi}$; temperature (TEM), $600^{\circ} \mathrm{C}$; ion spray Voltage floating (ISVF), $5500 \mathrm{~V}$ or $-4500 \mathrm{~V}$, in positive or negative modes, respectively; declustering potential (DP), $60 \mathrm{~V}$ or $-60 \mathrm{~V}$ in positive or negative modes. and collision energy for Product lon, $35 \mathrm{eV}$ or $-35 \mathrm{eV}$, in positive or negative modes, respectively; collision energy spread (CES), $15 \mathrm{eV}$. ESI source parameters on TripleTOF 6600 were changed as followings: ion source gas 1 (GS1), 40 psi; ion source Gas 2 (GS2), 80 psi; curtain gas (CUR), $30 \mathrm{psi}$; temperature (TEM), $500^{\circ} \mathrm{C}$; ion spray Voltage floating (ISVF), $5000 \mathrm{~V}$ or $-4000 \mathrm{~V}$, in positive or negative modes, respectively; other parameters were set as the same as 
TripleTOF 5600.

Data acquisition and data analysis. The LC-MS analyses were performed using a UHPLC system (1290 series, Agilent Technologies) coupled to a quadruple time-of-flight mass spectrometer (AB Sciex TripleTOF 5600 or 6600). Detailed parameters are provided in Supporting Information. For SWATH acquisition, the cycle time was set as $1 \mathrm{~s}$, including one TOF-MS scan (50 ms/scan; mass scan range 50-1050 Da) and 20 SWATH-MS ${ }^{2}$ scans (45 ms/scan; 50 Da/SWATH window; mass scan range 25-1050 Da). Therefore, 20 SWATH scans cover the mass range 50-1050 Da. All of the SWATH-MS ${ }^{2}$ spectra $^{-1}$ were acquired in high sensitivity mode. For $\mathrm{MS}^{2}$ acquisition, collision energy was ramped from 15 to 45 $\mathrm{eV}$ and charge state was set as 1. In standard DDA mode, general parameters, such as cycle time, mass range were set as the same as SWATH acquisition. Several other DDA specific parameters were setting as following: top 20 most intense ions (number of $\mathrm{MS}^{2}$ scan); minimum precursor ion intensity (200 cps), exclude former target ions (8 seconds after 2 occurrences), isolation window (unit resolution, $0.7 \mathrm{Da})$, and dynamic background subtraction applied.

For data analysis, First, data files in $\mathrm{mzXML}$ format were imported into $\mathrm{R}$, and peak detection was operated on $\mathrm{MS}^{1}$ data. CentWave algorithm developed by Tautenhahn et al. and included in XCMS, is used for peak detection. In peak detection, parameter "peakwidth" is set as $(5,30)$ in unit of seconds, referring to the range of minimum and maximum values of peak width in seconds. Please note that the parameter set "peakwidth" is LC condition dependent (such as column and flow rate). Users can adjust the "peakwidth" value according to the LC conditions. Parameter "sn", referring to signal to noise ratio, is set as 6 .

Library construction. In our experiment, to obtain accurate identification, the acquisition conditions of spectral library (including instrument type and collision energy level) are consistent with experiment data. Firstly, the 786 metabolites MS/MS spectral library was constructed using commercial chemical standards. Targeted product ion scan was applied on each metabolite standard solution to acquire the MS/MS spectra raw data. Then we averaged at least $11 \mathrm{MS} / \mathrm{MS}$ spectra data of each metabolite to generate one consensus MS/MS spectrum with good reproducibility. The consensus spectrum strategy to improve spectral reproducibility followed the instruction in a recent publication by NIST. ${ }^{2}$ Only five most abundant fragment ions of each metabolite were selected to generate MetDIA library. Compared with low-intensity ions, intensive fragment ions are more robust to resist the inference or contaminant in biological samples. From our experience, five fragment ions is enough to identify the metabolite in our 786 metabolite library. Adding additional fragment ions for targeted extraction is feasible in MetDIA package. The current library contains 786 metabolites in total, 765 for positive mode and 757 for negative mode. 
MetDIA also supports user to import their own libraries from a .csv file. The demo .csv file was provided as attachment, "Demo spectral database.csv". The format of .csv file must keep consistent with the demo file, and case matters for the column names. In this step, users can determine how many fragment ions one metabolite contains.

The $1^{\text {st }}$ column, "PrecursorMz", is the $\mathrm{m} / \mathrm{z}$ of precursor ion.

The $2^{\text {nd }}$ column, "ProductMz", is the $\mathrm{m} / \mathrm{z}$ of product ion.

The $3^{\text {rd }}$ column, "Retention.time", is the retention time. "NA" means unavailable time.

The $4^{\text {th }}$ column, "LibraryIntensity", is the relative intensity of product ions.

The $5^{\text {th }}$ column, "Name", is the index or the name of metabolites. The name must be unique for each metabolite. We highly recommend that use the index, such as "L0014_[M+H]+". Some character, such as "/", "I", "?" may cause errors during data processing.

$\mathrm{R}$ code is provided in "Demo spectral database.R", users could generate their specific spectral libraries use the code.

Culture of ${ }^{13} \mathrm{C}$ fully labeled bacteria. ${ }^{13} \mathrm{C}$ labeled M9 median is composed of $1 \mathrm{~mol} / \mathrm{L} \mathrm{MgSO}_{4}(400$ $\mu \mathrm{L}), 1 \mathrm{~mol} / \mathrm{L} \mathrm{CaCl} 2(100 \mu \mathrm{L}),{ }^{13} \mathrm{C}$ fully labeled glucose $(0.8 \mathrm{~g}), \mathrm{H}_{2} \mathrm{O}(100 \mathrm{~mL})$, and $\mathrm{M} 9$ aqueous solution (2X, $100 \mathrm{~mL})$. $\mathrm{M} 9$ aqueous solution (2X) is composed of $\mathrm{Na}_{2} \mathrm{HPO}_{4}(6.8 \mathrm{~g}), \mathrm{KH}_{2} \mathrm{PO}_{4}(3 \mathrm{~g}), \mathrm{NaCl}(0.25 \mathrm{~g})$, $\mathrm{NH}_{4} \mathrm{Cl}(0.5 \mathrm{~g})$, and $\mathrm{H}_{2} \mathrm{O}(400 \mathrm{~mL})$. First add $E$. coli BL21 codon plus to $4 \mathrm{~mL}{ }^{13} \mathrm{C}$ labeled $\mathrm{M} 9$ median containing chloromycetin $(30 \mu \mathrm{g} / \mathrm{mL})$ and culture overnight with shaking at $37^{\circ} \mathrm{C}$ and $220 \mathrm{rpm}$. Then, take $100 \mu \mathrm{L}$ aliquot from previous step and incubate with $4 \mathrm{~mL}{ }^{13} \mathrm{C}$ labeled $\mathrm{M} 9$ median containing chloromycetin $(30 \mu \mathrm{g} / \mathrm{mL})$, and culture overnight with shaking at $37^{\circ} \mathrm{C}$ and $220 \mathrm{rpm}$. And repeat this dilution and incubation three times in total to ensure complete ${ }^{13} \mathrm{C}$ labeling of bacteria. ${ }^{3}$ After that, transfer $4 \mathrm{~mL}$ aliquot of ${ }^{13} \mathrm{C}$ labeled bacteria into $180 \mathrm{~mL}{ }^{13} \mathrm{C}$ labeled $\mathrm{M} 9$ median containing chloromycetin $(30 \mu \mathrm{g} / \mathrm{mL})$, and grow with shaking at $37{ }^{\circ} \mathrm{C}$ and $220 \mathrm{rpm}$ to $\mathrm{OD}_{600}=1.6$. Finally, fully ${ }^{13} \mathrm{C}$ labeled bacteria is harvested for metabolomics analysis.

Score combination using ${ }^{13} \mathrm{C}$ labeled bacteria. Unlike proteomics, in which optimization and combination of score system is well established due to the decoy database. Ineffective decoy database in metabolomics leads that empirical knowledge-based determination. But, here we used two different approaches, standard mixture and ${ }^{13} \mathrm{C}$ fully labeled extracts, to prove that PPC, SSM and their combination are effective enough to separate the real metabolites from accidently constructed peak groups (Figure 3b, 3d).

The score distributions of 30STD_mix (Figure 3b) and ${ }^{13} \mathrm{C}$ fully labeled extracts (Figure S4a) also demonstrate that PPC performs better classification than SSM. Receiver operating characteristic analysis provides the same result that AUC of PPC (0.976) is higher than SSM (0.955) (Figure 3c). We 
then tested different combinations of PPC and SSM (Figure S4b). The result shows that the number of false positive identification is relatively low when the percentage of PPC is more than 0.3.

Comparison with targeted approaches in Proteomics. Current SWATH data processing programs are mostly developed and optimized for proteomics (like OpenSWATH, and AB Sciex $\left.S W A T H^{T M}\right)$. We found these tools are not effective for metabolite identification in metabolomics. Here, we choose $A B$ Sciex SWATH ${ }^{\mathrm{TM}}$ (a software plugin in Peakview to process SWATH data) and OpenSWATH to process metabolomics data, and demonstrate their poor performance on metabolite identification (Figures S1 and S2 in the Supporting Information).

We first demonstrated the use of AB Sciex SWATH ${ }^{T M}$ to process 30STD_mix data. We input the information of 30 metabolites to construct a library for targeted extraction. For each metabolite, we gave a simulated peptide sequence. Then SWATH ${ }^{\mathrm{TM}}$ considers each metabolite as a target to extract its corresponding peak group of fragment ions. In this step, however, SWATH ${ }^{\mathrm{TM}}$ program sometimes extracts the baseline rather than the correct peak groups. 8 out of 30 metabolites' peak groups (or $26.7 \%$ of metabolites) were wrongly assigned to the baseline. Two example are given in Figure $\mathrm{S} 1$ in the Supporting Information. The reason for wrong assignments is unknown. For metabolite identification, $\mathrm{SWATH}^{\mathrm{TM}}$ requires peptide sequence to generate decoy database. Since metabolite does not have a sequence, the metabolite identification is not feasible to achieve using SWATH ${ }^{\mathrm{TM}}$. Therefore, SWATH ${ }^{\mathrm{TM}}$ could only extract and generate peak groups with metabolomics data. However, some generated peak groups sometimes are mistakenly assigned with unknown reason.

Secondly, we have tested another software, OpenSWATH, for metabolomics data processing. Here, we input the library containing 765 metabolites as the target library. To construct a decoy database for metabolite identification, we adopted a mass shift approach from previous published MRMPROB program (Anal. Chem, 2013, 85, 5191-5199), which randomly subtracts 2 5 Da from fragment ions. We submitted both target library and decoy library to OpenSWATH for data processing. Without the restriction of precursor ions, OpenSWATH extracts peaks groups of 742 target metabolites and 721 decoy metabolites. With 1\% FDR defined by decoy database, 25 out of 30 metabolites could be identified in 30STD_mix. However, 241 false positive metabolites were also identified (Figure S2 in the Supporting Information). We further calculated the score ranking of 30 metabolites in MetDIA and OpenSWATH. 30 real metabolites in MetDIA ranked as top 35 metabolites, but their ranking in OpenSWATH distributed from top 4 to top 477. Therefore, OpenSWATH software, one of the widest used software in proteomics, is not suitable for metabolomics, which generates high false positive identifications of metabolites.

Again, we have to emphasize that, both programs are excellent for proteomics data processing, and 
not designed for metabolomics. It is reasonable that they do not have good performance for metabolite identification.

MS-DIAL parameters. The same DIA dataset processed by MetDIA were processed again using MS-DIAL according to the publication ${ }^{4}$ and user manual (http://prime.psc.riken.jp/Metabolomics_Software/MS-DIAL/index.html). The MS/MS spectral library in MS-DIAL program was also replaced by our in-house library (786 metabolites). For fair comparison, we used the same (or similar) parameters in MetDIA and MS-DIAL, specified as following: minimum peak width (5 scan); range of retention time (0-20 min); mass range (50-1050 Da); centroid data conversion (tolerances for MS1 and MS2 data, 0.01 Da and 0.014 Da, respectively). Metabolite identification parameters were set as following: retention time tolerance $100 \mathrm{~min}$ (referring to no restriction on retention time); accurate mass tolerance (MS1) $0.01 \mathrm{Da}$; accurate mass tolerance (MS2) $0.04 \mathrm{Da}$; identification score cut off $80 \%$. Peak detection parameter, "minimum peak height", was optimized among 500, 1000, 2000, 3000, 4000. 3000 was selected because of its relative high sensitivity and specificity (Figure S6). Other parameters for data processing in MS-DIAL program were set as the recommended values provided by manual. The detailed parameters are listed below:

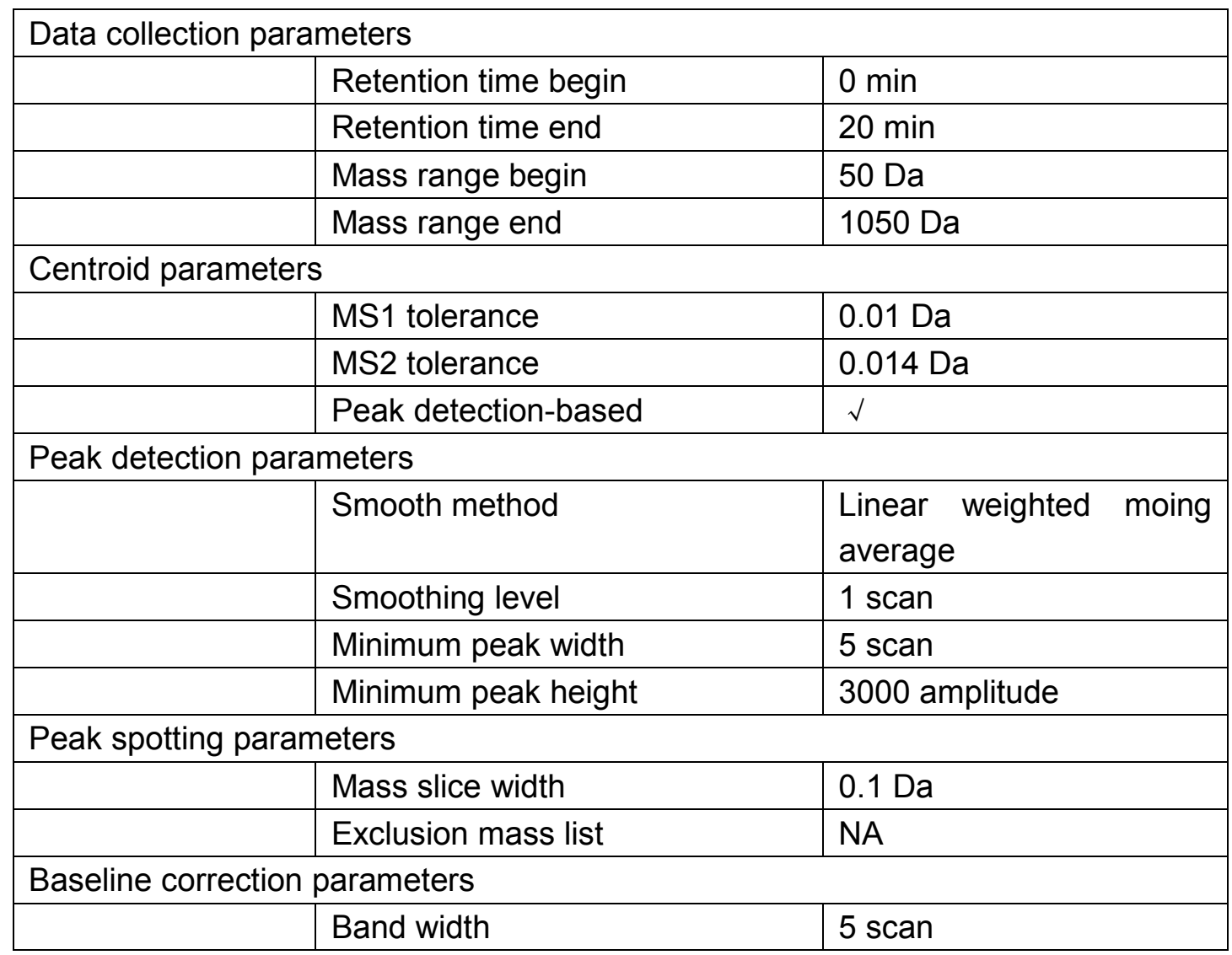




\begin{tabular}{|c|c|c|}
\hline & Segment number & 1 \\
\hline \multicolumn{3}{|c|}{ Deconvolution parameters } \\
\hline & Peak consideration & Both \\
\hline & Sigma window value & $0.001 \mathrm{~min}$ \\
\hline \multicolumn{3}{|c|}{ Purification parameters } \\
\hline & MS/MS abundance cut off & 0 amplitude \\
\hline & Exclude after precursor & $\sqrt{ }$ \\
\hline \multicolumn{3}{|c|}{ MSP file and MS/MS identification setting } \\
\hline & MSP file & $\begin{array}{l}\text { Library containing } 765 \\
\text { metabolites }\end{array}$ \\
\hline & Retention time tolerance & $100 \mathrm{~min}$ \\
\hline & Accurate mass tolerance (MS1) & $0.01 \mathrm{Da}$ \\
\hline & Accurate mass tolerance (MS2) & $0.014 \mathrm{Da}$ \\
\hline & Identification score cut off & $80 \%$ \\
\hline \multicolumn{3}{|c|}{ Alignment parameters setting } \\
\hline & Retention time tolerance & $0.1 \mathrm{~min}$ \\
\hline & MS1 tolerance & $0.025 \mathrm{Da}$ \\
\hline & Retention time factor & 0.5 \\
\hline & MS1 factor & 0.5 \\
\hline & Peak count filter & $0 \%$ \\
\hline
\end{tabular}

Analysis for Jurkat cell samples. Metabolites identified in both positive and negative mode were combined. $\mathrm{MS}^{1}$ peak areas for each metabolite in Jurkat cell samples were first normalized with total intensity. Then fold change was calculated with mean values from WT and FADD deficient groups, and p-value was also calculated with Welch $t$-test between two groups. Metabolites with fold change larger than 1.5 and $p$-value less and 0.01 were considered as significantly changed ones. These metabolites were submitted to pathway enrichment analysis using MetaboAnalyst. ${ }^{5}$

\section{Reference}

(1) Cai, Y.; Weng, K.; Guo, Y.; Peng, J.; Zhu, Z.-J. Metabolomics 2015, 11, 1575-1586.

(2) Yang, X.; Neta, P.; Stein, S. E. Anal Chem 2014, 86, 6393-6400.

(3) Bennett, B. D.; Yuan, J.; Kimball, E. H.; Rabinowitz, J. D. Nature Protocols 2008, 3, 1299-1311.

(4) Tsugawa, H.; Cajka, T.; Kind, T.; Ma, Y.; Higgins, B.; Ikeda, K.; Kanazawa, M.; VanderGheynst, J.; Fiehn, O.; Arita, M. Nat Methods 2015, 12, 523-526.

(5) Xia, J.; Psychogios, N.; Young, N.; Wishart, D. S. Nucleic Acids Res 2009, 37, W652-660. 